\title{
THE
}

1979

\section{Quantum Effects in the Spin Dynamics of the Linear Heisenberg Antiferromagnet}

Gerhard Müller

University of Rhode Island, gmuller@uri.edu

Hans Beck

Jill C. Bonner

University of Rhode Island

Follow this and additional works at: https://digitalcommons.uri.edu/phys_facpubs

Terms of Use

All rights reserved under copyright.

\section{Citation/Publisher Attribution}

Müller, G., Beck, H. \& Bonner, J. C.. Quantum effects in the spin dynamics of the linear Heisenberg antiferromagnet. J. Appl. Phys. 50 (1979), 7404-7406.

Available at: http://dx.doi.org/10.1063/1.326908.

This Article is brought to you for free and open access by the Physics at DigitalCommons@URI. It has been accepted for inclusion in Physics Faculty Publications by an authorized administrator of DigitalCommons@URI. For more information, please contact digitalcommons-group@uri.edu. 


\title{
Quantum effects in the spin dynamics of the linear Heisenberg antiferromagnet
}

\author{
Gerhard Müller ${ }^{\mathrm{a}}$ and Hans Beck ${ }^{\mathrm{a}}$ \\ Institute for Theoretical Physics, University of Basel, Basel, Switzerland \\ Jill C. Bonnerb, c \\ University of Rhode Island, Kingston, R.I. 02881
}

We present an approximate analytic expression for the dynamical spin correlation function of the $S=1 / 2$ linear Heisenberg antiferromagnet at $T=0$. The basis for our approach is that in zero field the spectrum is dominated by a double continuum [in $(q, \omega)$-space] of triplet spin wave excitations. The $S=1 / 2$ integrated intensity agrees very well with recent neutron scattering results on CPC, unlike the corresponding classical intensity. Moreover, the $S=1 / 2$ spectral weight function shows increasing asymmetry as $\mathrm{q} \rightarrow \pi$, a quantum effect, observable in more recent neutron scattering data. In non-zero magnetic field, there exist two, partly overlapping, double continua, each giving rise to a peak situated at the lower boundary. The ( $\mathrm{zz}$ component of) spectral weight function therefore has a double-peaked structure, as observed experimentally. Theory and experiment are in apparent agreement concerning the energy difference between the peaks.

PACS numbers: $75.40 . \mathrm{Fa}, 75.30 . \mathrm{Ds}, 75.10 . \mathrm{Jm}$

\section{INTRODUC'TION}

Spin dynamical theories ${ }^{1}$ of quasi-one-dimensional magnetic systems have relied heavily on the classical ( $\operatorname{spin} S=\infty$ ) Heisenberg linear chain. A very good reason for this is that the classical model is exactly solvable, for both the static thermodynamic properties and the correlation functions, in zero applied magnetic field ${ }^{2}$, and reliable and very comprehensive numerical calculations are available for non-zero applied field ${ }^{3}$. However, there has recently been a growing appreciation of the importance of quantum effects at very low temperatures, especially now that experimental techniques are sufficiently refined to allow reliable investigation of such subtleties 4 . Unfortunately, the $S=1 / 2$ (extreme quantum limit) Heisenberg linear chain remains an intractable analytic problem as far as finite temperature thermodynamic properties and spin correlation functions are concerned ${ }^{5}$. Extensive numerical studies exist for the static properties of the $S=1 / 2$ Heisenberg chain 6 and also for $S \geq 1$ Heisenberg chains ${ }^{7}$. However, little reliable information is available on the spin-spin correlations, and hence the spin dynamics, of non-classical chains. This report surveys a recent attack on the $S=1 / 2$ antiferromagnetic problem using a combination of theoretical techniques. Our information is derived from (a) exact finite chain calculations on systems of up to 10 spins; (b) exact selection rules which show which classes of states have non-zero matrix elements with the ground state (and hence contribute to the spin dynamics); (c) from exact sum rules; and (d) from both exact and approximate dispersion curves. We feature an analytic expression for the dynamical correlation function in $(q, w)$-space derived using the above information. Our expression is not exact since it violates exact sum rules (albeit by a small amount), but it yields good agreement with the few known exact results for the $S=1 / 2$ chain. Further our study reveals an unexpected richness and complexity of behavior which must be taken into account in attempting a fully rigorous treatment. It allows for the first quantitative interpretation of recent neutron scattering experiments in zero field 8 and a field of 70 $\mathrm{kOe}^{9}$. The quantum effects observed in a non-zero applied magnetic field are particularly striking. We hope to extend this approach to quantum chains with $S>1 / 2$, motivated by recent neutron scattering experiments showing an anomalous feature of the excitations of the $S=5 / 2$ linear chain TMMC in a field of $70 \mathrm{kOe} 10$.

\section{DISPERSION SPECTRA}

Fig. 1 is a sketch of the dominant low-lying excitations of antiferromagnetic Heisenberg chains as a function of wave-vector $q$ in zero field. The dashed curve is the well-known Anderson spin-wave (equivalent to classical $S=\infty$ ) disnersion relation, given by $E(q)=$ $\mathrm{J}|\sin \mathrm{q}|^{11}$, for a Hamiltonian $\mathrm{H}=\mathrm{J}_{i=1} \sum_{1}^{N} \overrightarrow{\mathrm{S}}_{1} \cdot \overrightarrow{\mathrm{S}}_{i+1}$. For the case $S=1 / 2$, on the other hand, the des cloizeaux and Pearson triplet excitations whose dispersion relation is $E_{1}(q)=\frac{\pi J}{2}|\sin q|^{12}$ form the lower boundary of a triplet spin-wave double continuum (SWDC) in $(q, \omega)$-space. The upper boundary may be calculated exactly by an extension of $\mathrm{dCP}$ techniques to be $\mathrm{E}_{2}(\mathrm{q})=\pi \mathrm{J}|\mathrm{sinq} / 2| 13$, 15 Our finite chain calculations show that all the states of the SWDC sketched in Fig. 1, have non-zero spectral weight, in accordance with earlier sum rule calculations of Hohenberg and Brinkman 14 , preliminary finite chain calculations 15 , an approximate semi-classical calculation 16 and a dynamical Hartree-Fock calculation ${ }^{17}$. This distinction between the excitations which are dominant for $S=\infty$ and $S=1 / 2$ has important consequences for the low temperature dynamical correlation function $S(q, \omega)$, which is the spacetime Fourier transform of the unequal time spin-spin cor-

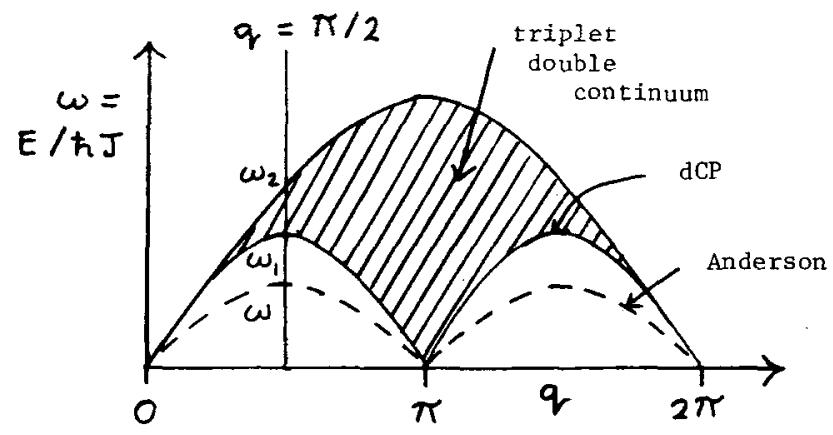

Fig. 1 The dominant low-lying excitations with non-zero spectral weight for the Anderson classical chain (dashed curve) and the $S=$ $1 / 2$ quantum chain (double continuum, shown shaded. 


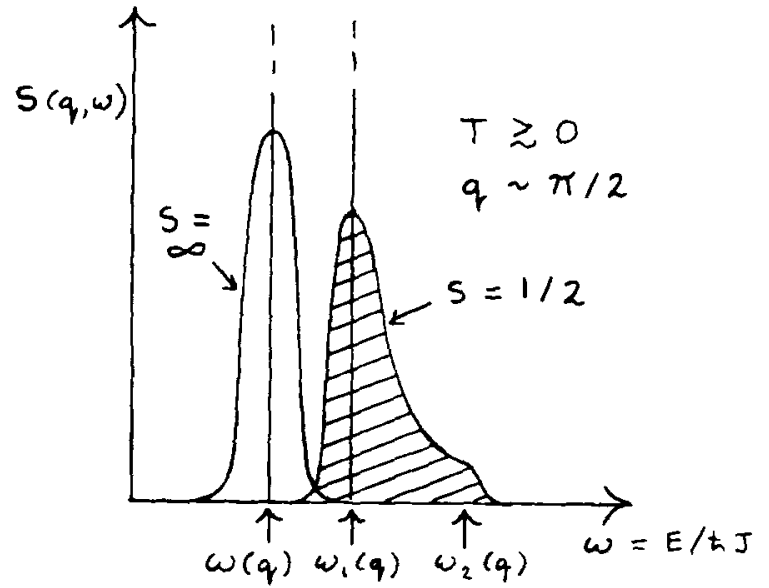

Fig. 2 The spectral weight function, $S(q, \omega)$ vs. $\omega$ at very low temperature for the classical chain (narrow symmetric peak) and the quantum chain (broader asymmetric structure).

relation function $\left\langle\vec{S}_{\ell}(t) \cdot \vec{S}_{\ell},(0)\right\rangle$. It has been shown that in the classical case, all the spectral weight is concentrated at the Anderson frequency, as sketched in Fig. 2. (The narrow peak becomes a delta function as $\mathrm{T} \rightarrow 0$ ). For the case of $S=1 / 2$, the bulk of the spectral weight occurs at the lower boundary, $E_{1}(q)$, but a tail persists to the upper boundary $E_{2}(q)$, also sketched in Fig. 2 . This feature will be discussed more specifically in section 4 .

\section{SELECTION RULES}

Finite chain calculations ${ }^{15}$, corroborated by exact analytic work 18 , reveal the existence of a second double continuum for $S=1 / 2$ and zero field. This is a singlet $(S=0)$ continuum, which becomes degenerate with the $S=1 \mathrm{SWDC}$ in the thermodynamic limit, i.e. in this limit, each state has a four-fold degeneracy. It is thus important to know which states have non-zero matrix elements with the ground state and therefore possess non-zero spectral weight. (For the classical chain, no such problem exists: only the Anderson states carry spectral weight, as noted above.) The situation becomes more complicated when a field $H$ is applied, since the ground state is no longer a singlet. Fortunately, we have proved exact selection rules which give a general answer to this problem. If $|\lambda\rangle$ are the eigenvectors of the Hamiltonian, the spin correlation functions involve matrix elements like $\left.<\lambda\left|S^{z}(q)\right| \lambda^{\prime}\right\rangle$; and $\left\langle\lambda\left|S^{ \pm}(q)\right| \lambda^{\prime}\right\rangle$, where the vector operator $\vec{S}(q) \equiv \frac{1}{\sqrt[N]{N}} \sum_{i=1}^{N} \vec{S}_{i}$. The eigenstate $|\lambda\rangle$ is characterized by good quanturn numbers wave-vector $k$; $z$ component of the total spin $S^{Z}$; and the total spin $S[S(S+1)]$. Selection rules on $k$ tell us that $\left\langle\left|S^{\mu}(q)\right|\right\rangle=0$ unless $q=k^{\prime}-k$, where $k$ and ' $k$ ' are the wave-vectors of the states $|\lambda\rangle$ and $\left|\lambda^{\prime}\right\rangle$. A selection rule relevant to the $z$ component of the dynamical correlation function, $S^{z Z}(q, \omega)$ is:

$\left\langle s^{z}(q) \mid\right\rangle=0$ unless the eigenstates $|\lambda\rangle$ and $\left|\lambda^{\prime}\right\rangle$ have identical $s^{z}$ values. For the transverse component, $\mathrm{S}^{ \pm}(\mathrm{q}, \omega)$, the relevant selection rule is $\left\langle\left|\mathrm{S}^{ \pm}(\mathrm{q})\right|\right\rangle=0$ unless the $S^{z}$ values differ by \pm 1 . For the total spin we have the important result that $\left\langle\left|S^{\mu}(q)\right|\right\rangle$ vanishes (a) unless the $S$ values of the constituent states differ by 0 or 1 , and (b) if both component states have $S=0$, i.e. are singlets. An important corollary of this total spin selection rule is that in zero field the states of the singlet SWDC carry no spectral weight; neither do lowlying states with $S \geq 215$. The only states which contribute are those of the triplet SWDC sketched in Fig. 1. In non-zero $H$ we have the novel result that two, partiy overlapping continua contribute, as sketched in Fig. 3. The two continua contain states which have the same $\mathrm{S}^{z}$. values as the (field-dependent) ground state, but whose $S$ values are either (a) the same as the ground state or

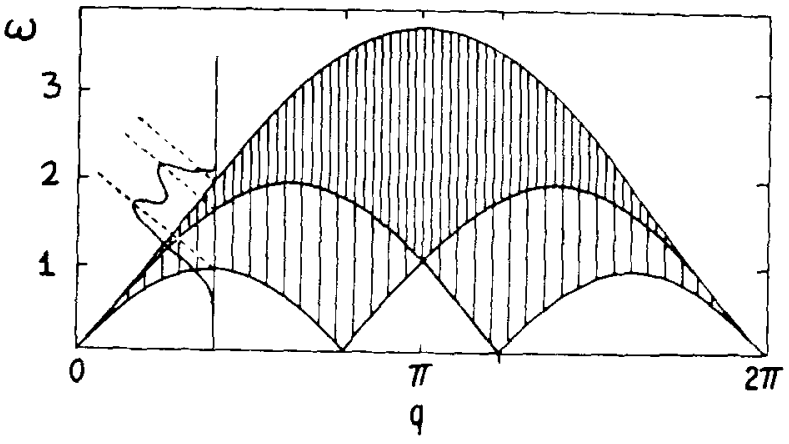

Fig. 3 Two, partly overlapping, double continua with a common upper boundary, for a field of $\mathrm{H}=$ $1 / 2 \mathrm{H}$ crit. The origination of the double peak in the spectral weight is shown superimposed.

(b) are greater by unity. The existence of two continua for $\mathrm{H}>0$ is corroborated by our finite chain calculations, and by studies based on work by Niemeijer on the exactly solvable $X Y$ mode1 19 . It should be noted that recent analytic work by Ishimura and $\mathrm{Shiba}$, which extends the dCP calculations to non-zero $\mathrm{H}^{20}$, is incomplete. The

Ishimura-Shiba calculation is indicated by the hatched line in Fig. 3 .

\section{EXPERIMENTAL COMPARISONS}

Our analytical expression for $S_{z W D C}(q, \omega)$, that part of the complete dynamical correlation function which originates from the SWDC, is

$$
\begin{gathered}
S_{\mathrm{zz}}^{\mathrm{SWDC}}(\mathrm{q}, \omega)=A\left[\omega^{2}-(\pi J / 2)^{2} \sin ^{2} q\right]^{-\frac{1}{2}} \Theta\{\omega-(\pi J / 2) \sin q\} \\
x \ominus\{\pi J \sin q / 2-\omega\},
\end{gathered}
$$

where $A$ is a constant. Exact sum rules exist for the susceptibility, energy, and integrated intensity. If the above approximate expression (I) is substituted into the sum rule integrals, values for $A$ are obtained which differ somewhat from each other, but suggest a "best value" of about 2. The reason is that we neglect some triplet states which exist outside the SWDC, and are very diffibut finite spectral weight in the thermodynamic limit. 21 Neglecting these states does not significantly affect the physics of the problem. Current experiment is quite insensitive to the exact value of $A$. At $T=0$, expression (1) has a divergence at $E_{1}(q)$, the lower boundary of the

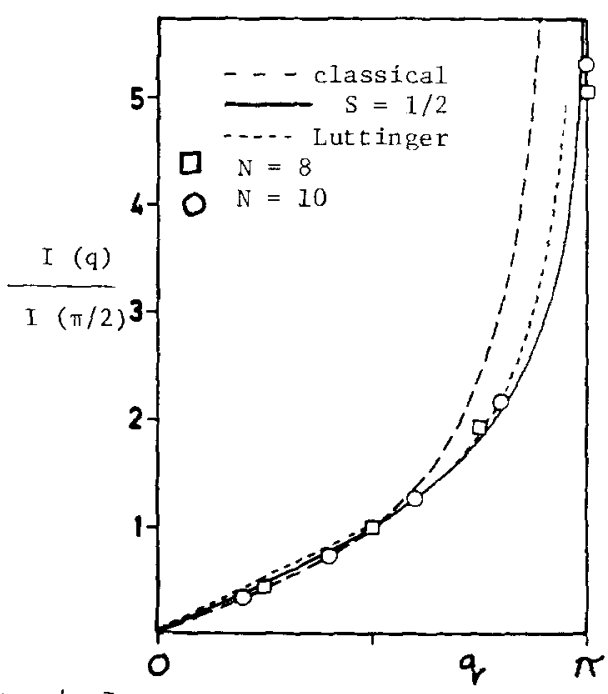

Fig. 4 Integrated intensity vs. wavevector is shown for three theoretical models. The curves are normalized to unity at $\mathrm{q}=\pi / 2$. 
SWDC, and a tail out to the upper boundary $E_{2}(q)$, where there is a cut-off. Thermal effects will produce the smeared-out version sketched in Fig. 2. The asymmetry pictured in Fig. 2 is not observable for small q, but becomes pronounced as $\mathrm{q} \rightarrow \pi$. This is a purely quantum effect which has now been observed experimentally.

A second quantity of experimental interest is the integrated intensity defined by

$$
I_{z z}(q)=\frac{1}{2 \pi} \int_{0}^{\infty} d \omega S_{z z}(q, \omega)
$$

Three theoretical calculations for $\mathrm{I}_{z z}(\mathrm{~g})$ are shown in Fig. 4. They are the classical result, ${ }^{8}$ a calculation based on the $S=1 / 2$ continum lattice (Luttinger) mode1 of Luther and Peschel ${ }^{22}$, and a result based on our approximate analytic expression (1). All curves are normalized to unity at $q=\pi / 2$. The classical curve diverges as $(\pi-q)^{-1}$ as $q \rightarrow \pi^{-}$, whereas our curve $I_{Z Z}^{\text {SWDC }}(q)$ diverges more weakly as $|\ln (\pi-q)|$. In Fig. 5, we make comparison with experimental results of Endoh et al. ${ }^{8}$. The experimental data points lie consistently below the classical curve, but are in quantitative agreement with our $S=1 / 2$ result out to $q \sim 0.8 \pi$. For still larger $q$, the situation is indeterminate because of the large error bars on the data.

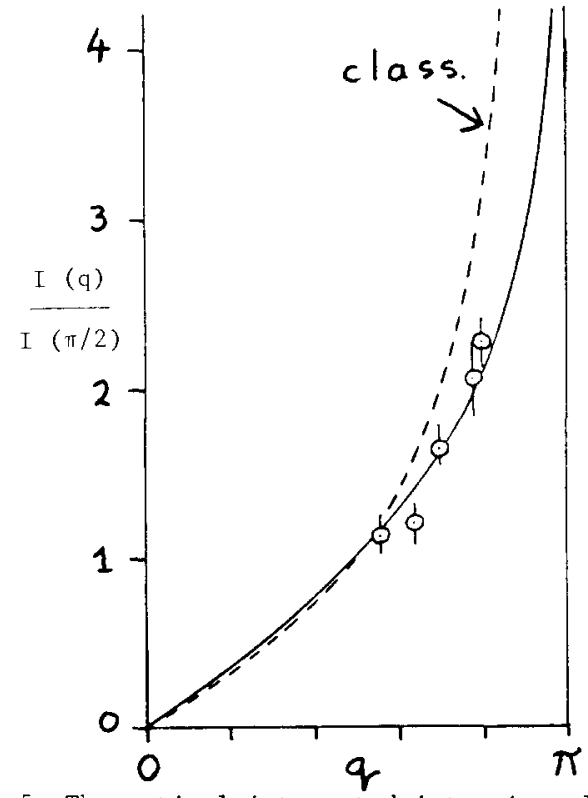

Fig. 5 Theoretical integrated intensity plotted in comparison with experimental data points from ref. 8. The data points fit our quantum curve much better than the classical curve for $0.5 \pi<q<0.8 \pi$. (Below $q=0.5 \pi$, the data fit either curve and are not shown).

Finally, we discuss the important implications of the $\mathrm{H}>0$ partly overlapping SWDC sketched in Fig. 3. Each continuum will have a $T=0$ divergence at its lower boundary, and a tail out to the common upper boundary. For low temperatures the two divergences will broaden out to give a double peak structure indicated in Fig. 3 . We therefore predict that low temperature neutron scattering will reveal double peak structure except at $q=\pi$ where the lower boundaries meet. Again, this is a dramatic quantum effect: The corresponding classical Heisenberg chain in a field behaves quite differently ${ }^{9}$. This double peak structure is ingeed observed in experiments on CPC in a field of $70 \mathrm{kOe}$. It should be noted, however, that our calculation is for $\mathrm{s}^{\mathrm{zZ}}(\mathrm{q},())$, the longitudinal component of the dynamical correlation function. In zero field $\mathrm{s}^{2 \mathrm{z}}(q, \omega)$ and the transverse component $\mathrm{s}^{ \pm}(q, \omega)$ are identical, but close to the antiferromagnetic critical field, the transverse component becomes dominant. The experiment under discussion ${ }^{9}$ appears to relate to $S^{ \pm}(q, \omega)$, a quantity we are currently calculating. However, since
70 kOe is a small fraction of the CPC critical field of about $400 \mathrm{kOe}$, we do not expect $\mathrm{S}^{\mathrm{zz}}(\mathrm{q}, \omega)$ and $\mathrm{s}^{ \pm}(\mathrm{q}, \omega)$ to differ significantly, and hence we can reasonably make comparison with experiment. Let us consider the theoretical continuum boundaries corresponding to a real field of $70 \mathrm{k0e}$. At the experimental value of the wave-vector $q$ the difference between the two lower boundaries, corresponding to the difference in energy between the double peaks, is $25 \%$ of the energy to the higher peak. This gives most encouraging agreement with experiment (Fig. 9 of ref. 9) which also corresponds to $25 \%$. Preliminary results for $S^{ \pm}(q, w)$ indicate the existence of yet a third peak which may not be readily observable at this relatively low field.

It would be interesting to repeat the neutron experiment with a different orientation of the CPC crystal, and to perform similar experiments on a crystal with a much lower critical field, e.g. a-CuNSal.

We are grateful for extensive and valuable discussions with Gen Shirane and John Axe, and, particularly, with Ian Heilman who helped supply both data and commentary

\section{REFERENCES}

(a) Supported by the Swiss National Science Foundation.

(b) Fellow of the Bunting Institute, Radcliffe College.

(c) Supported by NATO and the U.S. National Science Foundation, Grant No. DMR77-24136.

1. M. Steiner, J. Villain, and C.G. Windsor, Adv. Phys. 25, 87 (1976).

2. M.E. Fisher, Amer. J. Phys. 32, 343 (1964).

3. M. Blume, P. Heller, and N.A. Lurie, Phys. Rev. Bll, 4483 (1975)

4. R.J. Birgeneau and G. Shirane, Physics Today, p. 32, December 1978 .

5. J.C. Bonner, J. App1. Phys. 49, 1299 (1978) .

6. J.C. Bonner and M.E. Fisher, Phys. Rev. 135, A640 (1964).

7. H.W.J. Blöte, Physica 79B, 427 (1975).

8. Y. Endoh, G. Shirane, R.J. Birgeneau, P.M. Richards, and S.L. Holt, Phys. Rev. Lett., 32, 170 (1974).

9. I.U. Heilmann, G. Shirane, Y. Endoh, R.J. Birgeneau, and S.L. Ho1t, Phys. Rev. B 18, 3530 (1978).

10. I.U. Heilmann, R.J. Birgeneau, Y. Endoh, G. Reiter, G. Shirane, and S.L. Ho1t, Phys. Rev. B (to be published).

11. P.W. Anderson, Phys. Rev. 86, 694 (1952).

12. J. des Cloizeaux and J.J. Pearson, Phys. Rev. 128, 2131 (1962)

13. T. Yamada, Progr. Theor. Phys. 41, 880 (1969).

14. P.C. Hohenberg and W.F. Brinkman, Phys. Rev. B 10 , 128 (1974).

15. J.C. Bonner, B. Sutherland, and P.M. Richards, A.I.P. Conf. Proc. 24, 335 (1975).

16. H.J. Mikeska, Phys. Rev. B 12, 7 (1975); 12, 2794 (1975).

17. T. Todani and K. Kawasaki, Progr. Theor. Phys. 50, 4 (1973); 59, 1216 (1973).

18. J.D. Johnson, S. Krinsky, and B.M. McCoy, Phys. Rev. A 8, 2526 (1973)

19. Th. Niemeijer, Physica 36, 377 (1967).

20. N. Ishimura and H. Shiba, Progr. Theor. Phys. 57, 6 (1977); 57, 1862 (1977)

21. G. Muller and H. Beck, J. Phys. C 11,483 (1978).

22. A. Luther and I. Pesche1, Phys. Rev. B 12, 3908 (1975). 